\title{
Competencias investigativas en el personal académico de la Escuela de Ciencias de la Educación de la UNED, Costa Rica
}

\author{
Jensy Campos Céspedes, Linda Madriz Bermúdez, Olga Ligia Brenes Matarrita, Yarith Rivera Sánchez y \\ Marianella Viales Sossa \\ Universidad Estatal a Distancia, San Pedro Montes de Oca, San José, Costa Rica; jensycampos@gmail.com; Imadriz@uned.ac.cr; \\ obrenes@uned.ac.cr; yrivera@uned.ac.cr; mviales@uned.ac.cr
}

Recibido 17-I-2012 Corregido 31-V-2012 Aceptado 12-VII-2012

\begin{abstract}
Research Competences in the Academic Staff of the Education Sciences School of UNED-Costa Rica. Research is a relatively new emphasis in the Costa Rican Distance University, UNED. Here we present the results of a descriptive exploratory study on how the staff of the School of Education Sciences (ECE) performed self-evaluation with regard to their research competences. Based on the theoretical statements identified in the literature, a self-evaluation instrument was prepared and validated by four experts. The instrument was selfadministered by the academic faculty to all the ECE staff, with a $80 \%$ response (84 instruments). We corroborated the importance of taking into account the mastery of research competences in the processes of staff selection. Participants self-evaluated as weak in most competences that were included in the instrument, especially in aspects related with the technological skills, research management and the use of technological tools to support the research processes. It is important to orient the formative processes and the collaborative work towards the strengthening of those specific aspects.
\end{abstract}

\section{KEY WORDS}

Research competences, academic staff, teacher training, education research, Universidad Estatal a Distancia (Distance State University).

\section{RESUMEN}

Se presentan los resultados de un estudio exploratorio descriptivo, cuyo fin fue conocer cómo se autoevaluó el personal docente de la Escuela de Ciencias de la Educación de la UNED-Costa Rica (ECE) con respecto a su nivel de dominio de competencias para la investigación. Como parte del estudio se realizó una revisión de literatura sobre las competencias necesarias para la investigación en personal docente de educación superior. A partir de los planteamientos teóricos identificados en la revisión de literatura, se construyó un instrumento de autoevaluación el cual contó con una validación externa de cuatro expertos en el tema y que fue autoadministrado a un 100\% del personal académico de la ECE; con una tasa de respuesta del $80 \%$, correspondiente a 84 instrumentos. El estudio evidencia la importancia de tomar en cuenta el dominio de competencias para la investigación, en los procesos de selección del personal docente de la unidad académica. Asimismo, se observa que los participantes se autovaloran con debilidades en la mayor parte de las competencias que fueron incluidas en el instrumento; especialmente en aspectos relacionados con las habilidades tecnológicas, la gestión de la investigación y el uso de herramientas tecnológicas para apoyar los procesos investigativos. De allí la importancia de orientar los procesos formativos y el trabajo colaborativo hacia el fortalecimiento de esos aspectos específicos.

\section{PALABRAS CLAVE}

Competencias investigativas, personal académico, formación docente, investigación educativa, Universidad Estatal a Distancia.
Desde 1972, Stenhouse propuso la figura del docente investigador para definir al educador capaz de investigar sobre su propia práctica pedagógica y señaló la importancia de la investigación dentro de los procesos educativos (Stenhouse, 2003). Otros teóricos han insistido en la necesidad de que los procesos pedagógicos estén alimentados por los educativos y en que la investigación educativa sea una práctica inherente a la labor formativa en los sistemas de educación formal. Desde otras perspectivas más generales a la profesión docente, teóricos de la educación superior y del quehacer universitario plantean la investigación como piedra angular de la academia (De Miguel, 2003). 
En ambas vías, tanto desde la profesión docente, como desde la condición de académicos, quienes ostentan los cargos de tutores, encargados de Cátedra y de Programa de la Escuela de Ciencias de la Educación deben, necesariamente, incorporar el quehacer investigativo como parte de su labor. Para ello, más que la prescripción y la declaración de esa condición por parte de las instancias máximas de toma de decisión de la ECE, se requiere que cada académico desarrolle un conjunto de competencias que le permitan el ejercicio de la actividad investigativa y la incorporación de los resultados de las investigaciones en el mejoramiento de los procesos pedagógicos, así como el aporte a su campo disciplinar.

Desde lo anterior, es necesario que los esfuerzos para desarrollar la investigación en la ECE sean acompañados de procesos formativos para fortalecer las competencias investigativas en los académicos que forman parte de ella.

La revisión de literatura realizada y la profundización en el análisis de esta, permitió seleccionar otros perfiles de competencias investigativas propuestos por diferentes autores y hacer las adaptaciones para reelaborar un perfil propio de éstas que sirvan de base para orientar los procesos de selección y de formación del personal académico de la Escuela de Ciencias de la Educación de la Universidad Estatal a Distancia de Costa Rica.

En consecuencia, debido a que las competencias investigativas incluidas en el perfil son suficientemente genéricas para que el personal docente sea competente para el desarrollo de investigaciones, fácilmente este puede ser adecuado a cada institución educativa y servir como base y referente para la evaluación de competencias investigativas cuando se gestionen o diseñen procesos formativos en el campo de la investigación.

El perfil está constituido por cinco tipos de habilidades divididas en cuarenta y siete aspectos específicos, se pretende que el personal académico posea estos dominios para desarrollar los procesos de investigación (Fig. 1). Los cinco conjuntos de habilidades incluidos en el perfil son los siguientes:

- Habilidades cognitivas: constituyen el conjunto que permiten al profesional ser capaz de establecer las interacciones entre los elementos de una realidad. Estas destrezas ayudan a identificar las disonancias y los vacíos de información que requieren solventarse mediante la investigación para atender problemas de diversa índole relacionados con el objeto de estudio de la disciplina.

- Habilidades tecnológicas: involucran el dominio de diferentes herramientas tecnológicas que facilitan los procesos de búsqueda, sistematización, análisis e interpretación de las informaciones obtenidas mediante el proceso de investigación.

- Habilidades metodológicas: refieren al dominio de métodos, técnicas e instrumentos para la recolección y los análisis de información requeridos para responder a los problemas de investigación relacionados con el objeto de estudio de la profesión.

- Habilidades para gestionar la investigación: corresponden a las destrezas para identificar fuentes de financiamiento, captar y administrar eficazmente los recursos requeridos para el desarrollo de los proyectos de investigación.

- Habilidades para el trabajo en equipo: se relacionan con el conjunto de destrezas cognitivas, sociales y actitudinales que permiten desarrollar relaciones interpersonales armoniosas, enfocadas hacia el desarrollo del talento humano y la generación de conocimiento en el campo o la línea de investigación sobre la que se trabaja.

Ese perfil del docente investigador elaborado, es deseable para todos los académicos de la ECE, y básico para el personal docente que ostenta cargos de Encargado de Cátedra o de Programa, así como para los integrantes y asesores de la Comisión de TFG

Las debilidades por otra parte, en algunos de los aspectos incluidos en el perfil, brindarían información clave para orientar los procesos de formación continua del personal académico. Asimismo, el perfil de competencias investigativas permite a la institución enriquecer los procesos de selección del recurso humano, ya que las dependencias responsables de realizar los procesos de reclutamiento y selección del talento humano deberían incorporar ese perfil de competencias específicas dentro del perfil general que tiene el sistema de reclutamiento y selección del personal académico para garantizar que se capten los profesionales con las competencias que la institución requiere.

\section{METODOLOGÍA}

El objetivo principal de la investigación consistió en diagnosticar las competencias investigativas de los académicos de la ECE. Por eso, se realizó un estudio exploratorio descriptivo, organizado en dos fases. La primera fase consistió en una revisión de literatura, en la que se utilizó el análisis de contenido como principal técnica. Esta estrategia permitió analizar un número significativo de libros y artículos publicados en la red mundial, sobre competencias investigativas y derivar, desde los planteamientos de autores o estudiosos del tema, las competencias investigativas 


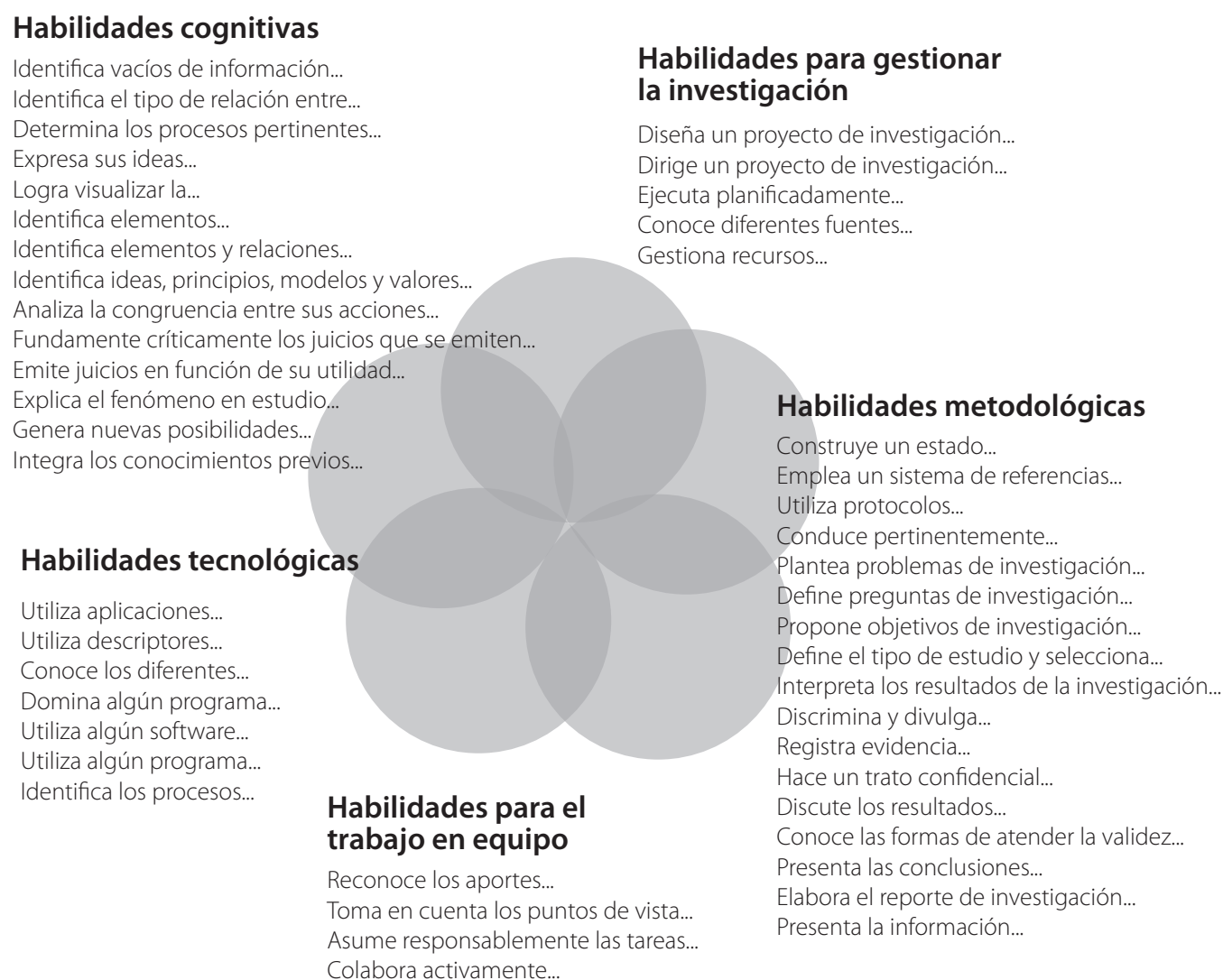

colabora activamente.

FIG. 1. Competencias claves en el perfil del personal académico formador de docentes.

claves deseables en un docente de escuela para que se desempeñe como investigador de su práctica pedagógica.

A partir de la revisión de literatura (Beneitone et al., 2007; Poblete \& Villa, 2008; Universidad Simón Bolívar, s.f.; Solanes et al., 2008; Campos \& Castro, 2010) se elaboró un perfil de competencias básicas que, con base en la teoría y la experiencia, debe poseer el personal académico de la ECE (Apéndice 1). El perfil se utilizó para la construcción de un instrumento de autoevaluación que fue aplicado al personal de la Escuela.

El instrumento fue sometido a validación externa por criterio de expertos, en este caso, cuatro personas que comparten características similares con la población en estudio. Además, el cuestionario se sometió a una prueba piloto que midió el tiempo de respuesta, la pertinencia y la claridad de los ítems incluidos y las instrucciones. Después de incorporar los cambios de la validación se obtuvo la versión final del instrumento y se procedió a la segunda fase del trabajo en la que se aplicó mediante la técnica de autoadministración e incluyó el 100\% del personal académico de la ECE (tutores, encargados de Cátedra, de Programa y de práctica profesional). La tasa de respuesta fue de $80 \%$, por lo que se contó con 84 instrumentos totalmente completos por el personal académico participante.

\section{RESULTADOS}

\section{Autoevaluación de las habilidades cognitivas}

Según los resultados obtenidos de la aplicación del instrumento, en la habilidad que el personal presenta mayores carencias es la que refiere a si "Logra visualizar la complejidad ante varias áreas de conocimiento sobre el objeto de estudio", mientras que las habilidades que concentran más cantidad de académicos que se consideran fuertes en esos aspectos son:

"Emite juicios en función de su utilidad, validez y confiabilidad a partir de la información analizada" e 
"Integra los conocimientos previos para el abordaje del fenómeno u objeto de estudio".

En términos generales, el personal académico de la ECE presenta mayor fortaleza en la capacidad para integrar los conocimientos previos y la emisión de juicios debidamente argumentados; sin embargo, la media para cada una de las habilidades cognitivas oscila entre 2,3 y 2,6, ello significa que la mayor parte del personal académico de la ECE autovalora su nivel de dominio de esas habilidades entre alto y muy alto (Fig. 2), lo que es una fortaleza muy importante en las funciones laborales de los académicos de la ECE.

\section{Autoevaluación de las habilidades tecnológicas}

En cuanto a la valoración que el personal académico hace respecto de sus habilidades tecnológicas, contrario al grupo de habilidades cognitivas, la mayor parte de los participantes considera tener bajo dominio de las tecnologías, específicamente, en cuanto al uso de software para procesar datos.

Pese a que este conjunto de competencias carece de una importancia preponderante, como sí la tienen las cognitivas, llama la atención el hecho de que exista bajo nivel de dominio en el personal académico cuando una de las líneas estratégicas de la institución universitaria se relaciona con el uso de las tecnologías aplicadas a la educación y uno de los puntos de lanza del plan estratégico de la Institución es la educación en línea y el desarrollo de la investigación. Asimismo, llama la atención que el personal académico presente bajo nivel de dominio en el uso de técnicas sofisticadas para la búsqueda y el acceso a fuentes especializadas, cuando éstas son habilidades indispensables que se deben formar en el estudiantado.

Siguiendo esta idea sobresale el hecho de que $60,7 \%$ de los participantes tiene bajo o ningún dominio de herramientas para detectar copia fiel de textos.

En términos generales el personal académico de la ECE presenta debilidades en cuanto a las habilidades tecnológicas necesarias para facilitar los procesos investigativos e incluso para favorecer la formación para la investigación del estudiantado a cargo. En este sentido, tal como se observa en la figura 3, de los tres puntos que representan el nivel alto de dominio, la media que se expresó por parte de los académicos es de 1,2 en cuanto a la utilización de herramientas para la detección de plagio, 1,5 para habilidades relacionadas con el uso de programas básicos para analizar datos y 1,7 en cuanto al uso de algún gestor bibliográfico. Es fundamental y un reto para todas las instancias involucradas de la UNED ofrecer diversas estrategias para lograr una mayor capacitación en este ámbito.

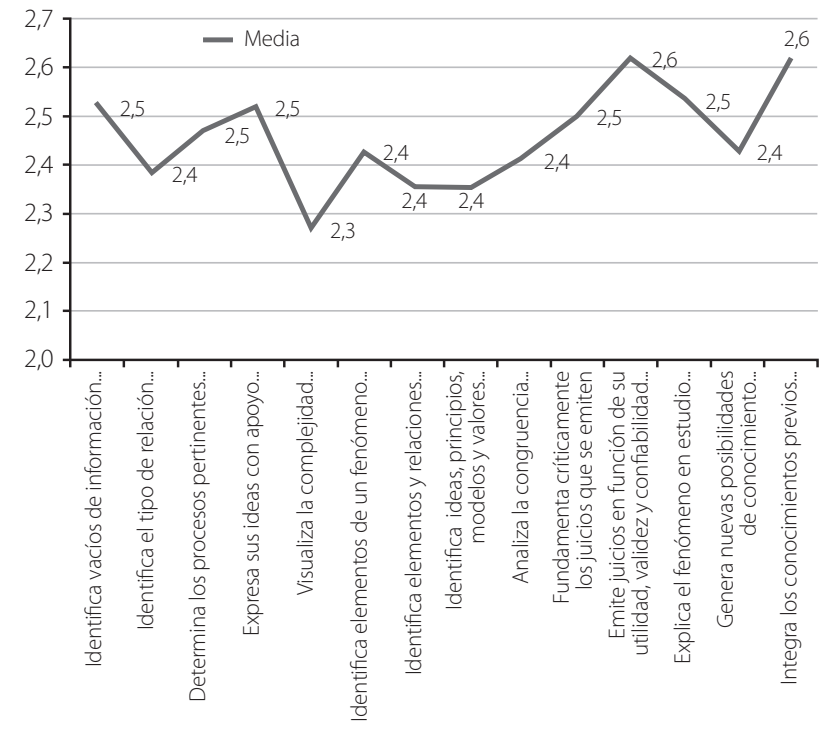

FIG. 2. Media de las habilidades cognitivas presentadas por el personal académico de la ECE, 2011.

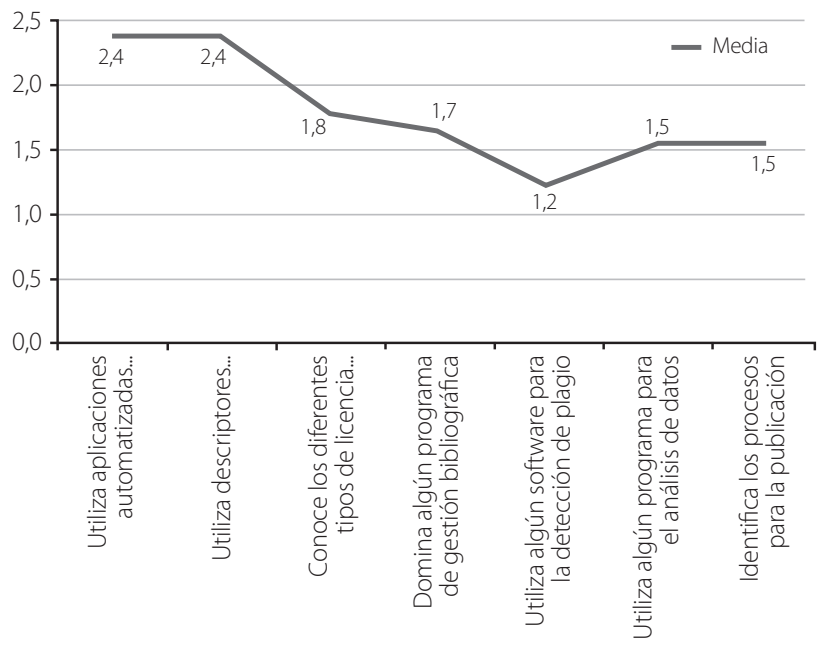

FIG. 3. Media presentada en la autoevaluación de los participantes respecto del dominio de sus habilidades tecnológicas (ECE, 2011). 


\section{Autoevaluación de las habilidades metodológicas}

En cuanto a las habilidades metodológicas, pese a que existe un mayor porcentaje de académicos que consideran que tienen un domino alto, existe un importante porcentaje de ellos que presentan dominio entre medio y nulo, sobre todo en aspectos relacionados con el uso de protocolos para garantizar la rigurosidad, el tratamiento ético y la utilización del consentimiento informado para los participantes de la investigación. Además, se observa bajo nivel de dominio en las habilidades relacionadas con las técnicas para asegurar la validez en los procesos investigativos, especialmente desde el enfoque cualitativo, la habilidad para la elaboración de informes y la divulgación de los procesos investigativos (Fig. 4).

\section{Autoevaluación de habilidades para la gestión de la investigación}

En cuanto a las habilidades para la gestión dentro del ámbito, el personal académico de la ECE presenta importantes debilidades, especialmente en lo referido al conocimiento de fuentes de financiamiento, la dirección de proyectos de este tipo y la gestión de recursos para la investigación. En este sentido, es importante y urgente

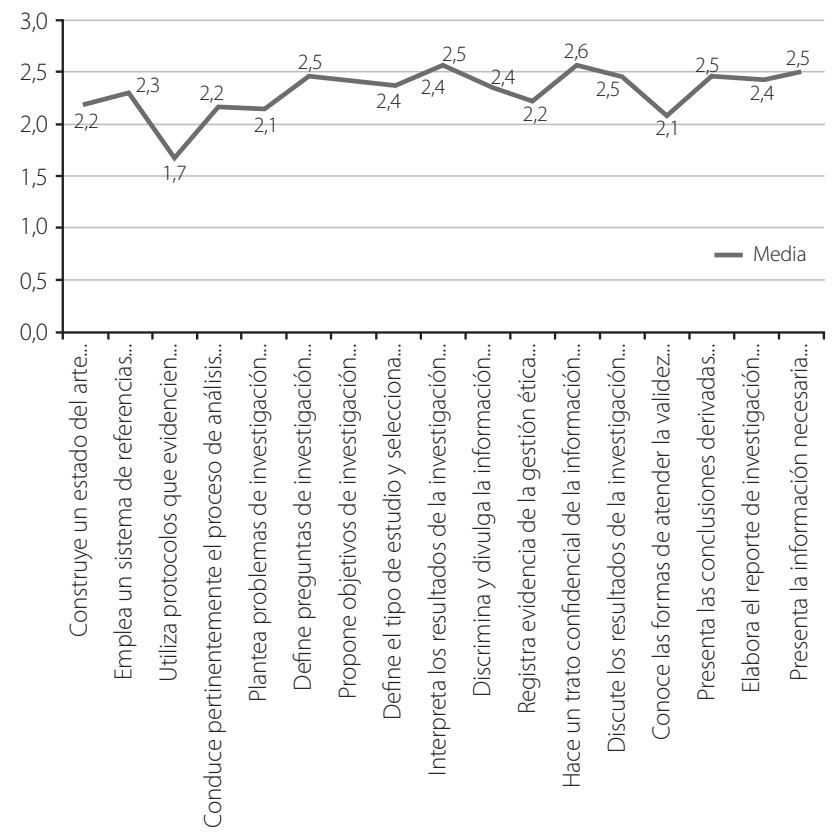

FIG. 4. Media presentada en la autoevaluación del personal académico de la ECE respecto de las habilidades metodológicas para el desarrollo de procesos investigativos (ECE, 2011). desarrollar acciones que permitan a los académicos desarrollar conocimientos, habilidades y destrezas para la identificación de fuentes de financiamiento y la captación de fondos para financiar proyectos de investigación (Fig. 5).

Ante las debilidades observadas en la figura 5 en torno a las habilidades para la gestión de la investigación, es importante que por parte de la ECE se desarrollen acciones formativas y ejercicios prácticos, mediante grupos de investigación, que permitan su fortalecimiento, ya que son clave para lograr, en el mediano y el largo plazo, hacer viable el desarrollo de la investigación en la Escuela y la generación de conocimiento, en especial en una coyuntura de limitación de recursos institucionales.

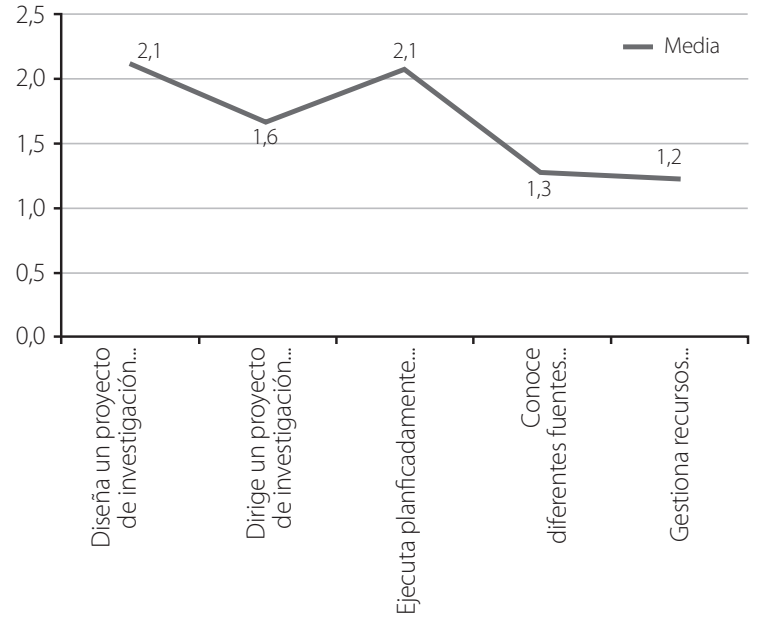

FIG. 5. Media presentada por el personal académico de la ECE en su autoevaluación respecto de las habilidades para gestionar la investigación (ECE, 2011).

\section{Autoevaluación de las habilidades para el trabajo en equipo}

La mayoría de los integrantes del personal académico incluido en el estudio se considera como altamente competente para el trabajo en equipo. Ello es positivo y podría explicar la relativa facilidad con la que se han ido constituyendo grupos de investigación en la ECE (Fig. 6).

De acuerdo con los resultados obtenidos se observa una brecha entre el dominio de las competencias investigativas que se desean para el personal académico de la ECE y los dominios que actualmente el personal tiene desde su propia perspectiva, tal como se observa en la figura 7. 


\section{DISCUSIÓN}

El tema de las competencias investigativas por parte del personal académico de la ECE remite, necesariamente, a una reflexión ontológica respecto del significado real de la academia y del quehacer del personal académico en una universidad y de lo que De Miguel (2003) denomina la responsabilidad socialmente conferida a la institución universitaria.

Es preciso posicionar a la investigación como proceso fundamental de todo el quehacer académico de los docentes, en cualquiera de los niveles donde se ubiquen,

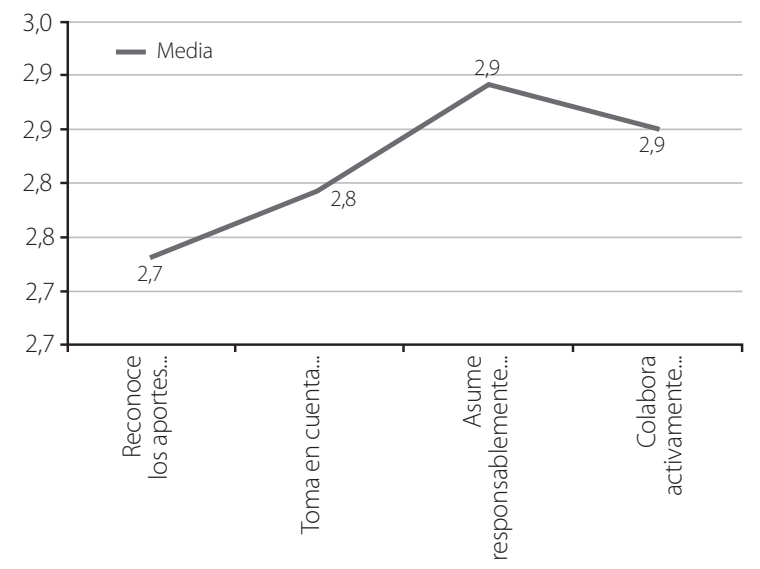

FIG. 6. Media presentada por el personal académico de la ECE en su autoevaluación respecto de las habilidades para el trabajo en equipo (ECE, 2011). pero especialmente en el ámbito de la Educación Superior. La función investigativa debe estar imbricada en el quehacer de los académicos. No se trata de que la ECE cuente con apoyo de investigadores, sino de que el personal académico esté formado por docentes investigadores. El posicionamiento de los docentes como investigadores de su quehacer, tal como lo plantea Stenhouse (2003), es lo que permite a la educación trascender y pasar de un quehacer a una disciplina científica.

La garantía de que el personal académico de la ECE esté formado por docentes investigadores le permite desarrollarse y trascender, pasar de unidad transmisora de conocimiento a unidad productora y gestora de conocimiento. Ello implica un cambio sustantivo en el quehacer, en el posicionamiento y en la proyección. También plantea una interacción distinta con las demás dependencias de la universidad y unos términos diferentes en aspectos tan concretos como la asignación de los recursos.

Parece que la academia en la UNED, desde su génesis, se ha caracterizado por ser unidad formadora que enfatiza los procesos formativos en la transferencia de contenidos consignados en una unidad didáctica. En palabras de Freire (Freire \& Faundez, 1986; Freire, 1987; 1997), podría calificarse como un trabajo desde la pedagogía de la respuesta y no desde una pedagogía de la pregunta, que sería lo que permite las mayores oportunidades de desarrollo de las potencialidades de los actores involucrados en los procesos de enseñanza y de aprendizaje, a la vez que abre las posibilidades de construir nuevos conocimientos.

Se trata, por lo tanto, de resignificar el quehacer académico de la ECE a partir de una nueva comprensión del

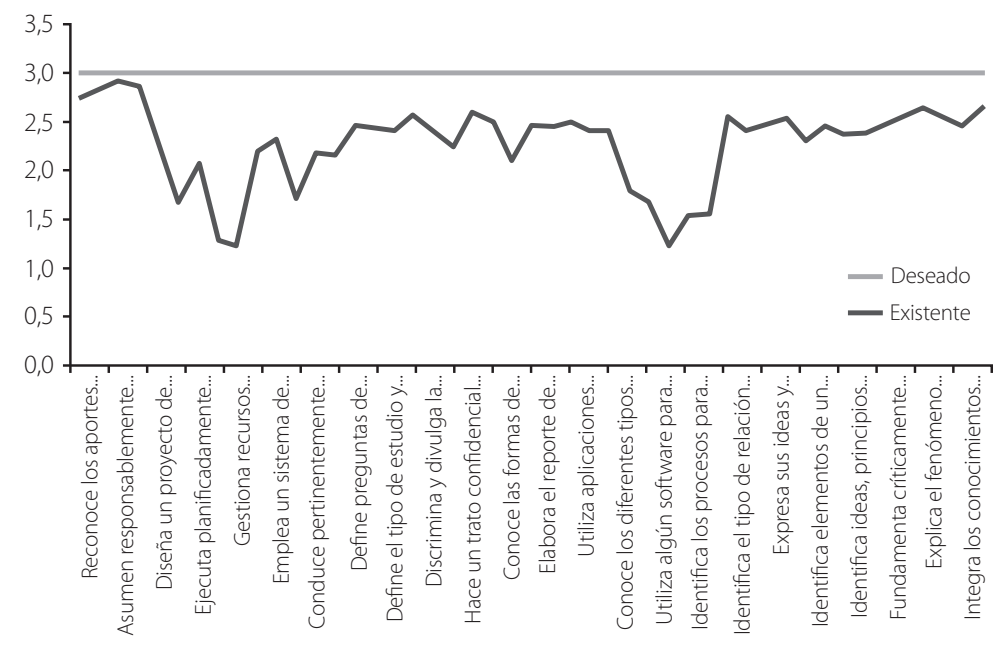

FIG. 7. Perfil deseado y perfil actual del personal académico de la ECE respecto de su capacidad para investigar (ECE, 2011). 
ser académico y ello, necesariamente, implica ubicar la investigación y la generación de conocimiento (y todas las acciones concretas que para ello se requieren) como parte imprescindible e innegable del ser académico.

La ECE requiere convertir su cuerpo académico en docentes investigadores. Eso significa, no que se van a convertir en investigadores (con la idea que posiblemente se tiene en el imaginario de lo que es un investigador), sino que son sujetos epistémicos capaces de producir conocimiento desde y en su práctica pedagógica y los encargados y encargadas de Programa (carrera) y de Cátedra deben poseer competencias que permitan la constitución de una comunidad de aprendizaje sostenible y en constante proceso de desarrollo hacia niveles cada vez más altos de calidad en cuanto a la generación y transferencia de conocimiento en el campo de la educación.

El instrumento utilizado evidenció ser un insumo muy útil para la recolección de la información deseada y reveló las áreas prioritarias por intervenir con las habilidades cognitivas, tecnológicas y metodológicas. Sin embargo, previo, es necesario el desarrollo de una reflexión y una discusión sobre los verdaderos fines de la ECE y los alcances que como academia se desean concretar en el mediano y largo plazo.

Ahora bien, los resultados presentados producto de la autoevaluación de las competencias investigativas, podrán brindar insumos convenientes para valorar la pertinencia de los procesos de formación continua que se están desarrollando con el personal académico de la ECE.

En ese sentido, es importante preguntarse si, de alguna manera, los procesos de formación y de capacitación desarrollados en la ECE más que enfocarse en las necesidades sentidas por el personal académico o por lo que resulta de la sumatoria de las opiniones del personal, conviene utilizar como referentes principales las metas institucionales en el mediano y largo plazo y en función de eso, haya que determinar los requerimientos de formación y el perfil académico apropiado para alcanzar esas metas a las que se aspira.

Asimismo, parece inevitable la pregunta acerca de los criterios de selección de alternativas de formación que desarrolla la universidad para elevar el nivel académico del personal docente, en este caso sería: ¿cuáles son las áreas temáticas seleccionadas para preparar al personal académico a nivel de doctorado y postdoctorado?

A partir del estudio realizado se detecta la necesidad de que la ECE elabore el perfil deseado para el personal académico y para cada uno de los puestos clave que requiere. Es necesario tener plena claridad del perfil requerido para los profesores, encargados de Cátedra, de Programa y asesores de práctica profesional y de trabajos finales de graduación. En este sentido, la elaboración del perfil deseado debe realizarse no solo con base en la normativa existente en la UNED, sino también en el plan de desarrollo institucional y en el plan de desarrollo estratégico de la Escuela, a la luz de las demandas y las necesidades sociales del país y de la región.

La construcción de ese perfil deseado o perfil de referencia para el personal académico de la ECE no solo permitirá mejorar los procesos de reclutamiento y de selección del personal académico, sino que puede orientar otras acciones estratégicas para el mejoramiento de la calidad académica de los programas, de los cursos y de las asignaturas; por ejemplo, la orientación para construir un banco de tutores con profesionales dispuestos a laborar para la institución y que cumplan con ese perfil.

Igualmente ese perfil de referencia se constituiría en un insumo clave para orientar la planificación y el desarrollo de un plan de formación del personal académico de la ECE en el que se articulen todos los procesos de formación continua y capacitación del personal académico de la ECE.

La determinación de un perfil del académico de la ECE resultante de investigación documental y del análisis de las necesidades de la Escuela, permitiría evaluar si los requerimientos establecidos en los procesos de reclutamiento y de selección se ajustan a esas necesidades y si las acciones formativas contribuyen a la formación de ese perfil requerido.

En el estudio realizado el análisis se circunscribió solamente a uno de los elementos que debe estar contenido en el perfil del académico de la ECE, cual es la capacidad para investigar. Se hace énfasis en esa competencia por la naturaleza genérica que esta competencia posee y por la preponderancia que la gestión del conocimiento asume en las sociedades modernas. En términos generales se puede decir que el personal académico de la ECE requiere fortalecer las competencias investigativas, especialmente, en los aspectos relacionados con las habilidades tecnológicas, la gestión de la investigación y el uso de herramientas tecnológicas para apoyar los procesos investigativos.

De igual manera, el estudio realizado devela la necesidad de potenciar el desarrollo de competencias investigativas en los formadores de docentes, como es el caso del personal académico de la ECE, por cuanto la investigación constituye un eje sustancial de la formación docente y la vía que puede permitir la generación de transformaciones, dada la complejidad de la sociedad y la crisis que se está viviendo en materia educativa y desde la Dirección de la ECE en coordinación con la COMI se trabaja en la propuesta de capacitaciones que faciliten el desarrollo de las habilidades detectadas como débiles. 


\section{REFERENCIAS}

Beneitone, P.C., Esquetini, J., González, M., Maleta, G., Siufi, R. \& Wagenaa, R. (2007). Tuning América Latina: Reflexiones y Perspectivas de la Educación Superior en América Latina (2004-2007). Bilbao, España: Universidad Deusto.

Campos J. \& Castro, C. (2010). El docente como investigador de su quehacer en Costa Rica. Patria Grande. San José, Costa Rica: CECC-SICA.

De Miguel, J. (2003). Universidad y Democracia. Cádiz: Fundación Municipal de Cultura Ayuntamiento de Cádiz. Cádiz, España: Ayuntamiento de Cádiz.

Freire, P. \& Faundez, P. (1985). Por una pedagogía de la pregunta. Río de Janeiro, Brazil: Paz e Terra.

Freire P. (1986). "Pedagogía de la pregunta". Río de Janeiro, Brazil: Paz e Terra.

Freire, P. (1997). Política y Educación. México: Siglo XXI.
Poblete, M. \& Villa, A. (2008). Aprendizaje basado en competencias. Una propuesta de evaluación de Competencias Genéricas. Bilbao, España: Ediciones Mensajero.

Solanes, A., Núñez, R. \& Rodríguez, J. (2008). Elaboración de un cuestionario para la evaluación de competencias genéricas en estudiantes universitarios. Apuntes de Psicología, 20(1): 35-49. Recuperado de http://www.cop.es/delegaci/andocci/files/contenidos/VOL_26_1_4.pdf

Stenhouse, L. 2003. La investigación y desarrollo del curriculum. Madrid, España: Morata.

Universidad Simón Bolívar. (s.f.). Instrumento para autoevaluación de habilidades y competencias para la investigación. Recuperado de www.usb.edu.mx/investigacion/cif/proyectos/.../propuesta.doc

Universidad Simón Bolívar (s.f.). Aplicación piloto del instrumento para la autoevaluación de competencias para la investigación. Recuperado de www.usb.edu.mx/investigacion/cif/ proyectos/.../informe.doc 


\section{APÉNDICE 1}

Escala de autoevaluación de habilidades para la investigación

\begin{tabular}{|c|c|c|c|c|}
\hline \multirow[b]{2}{*}{ CRITERIOS POR VALORAR } & \multicolumn{4}{|c|}{ NIVEL PERCIBIDO } \\
\hline & $\begin{array}{l}\text { Alto } \\
(3)\end{array}$ & $\begin{array}{l}\text { Medio } \\
(2)\end{array}$ & $\begin{array}{c}\text { Bajo } \\
(1)\end{array}$ & $\begin{array}{c}\text { Ninguno } \\
(0)\end{array}$ \\
\hline \multicolumn{5}{|l|}{ I. Habilidades cognitivas } \\
\hline $\begin{array}{l}\text { 1. Identifica vacíos de información o falta de coherencia en la argumentación en textos, } \\
\text { discursos y otras formas de comunicación. }\end{array}$ & & & & \\
\hline $\begin{array}{l}\text { 2. Identifica el tipo de relación entre los diversos elementos que constituyen el objeto de } \\
\text { investigación. }\end{array}$ & & & & \\
\hline $\begin{array}{l}\text { 3. Determina los procesos pertinentes para alcanzar los objetivos propuestos en } \\
\text { situaciones concretas. }\end{array}$ & & & & \\
\hline 4. Expresa sus ideas y conclusiones con apoyo en datos concretos. & & & & \\
\hline $\begin{array}{l}\text { 5. Logra visualizar la complejidad ante varias áreas de conocimiento sobre el objeto de } \\
\text { estudio }\end{array}$ & & & & \\
\hline $\begin{array}{l}\text { 6. Identifica elementos de un fenómeno u objeto de estudio y los diferentes tipos de } \\
\text { relación entre estos. }\end{array}$ & & & & \\
\hline $\begin{array}{l}\text { 7. Identifica elementos y relaciones para determinar convergencias y divergencias dentro } \\
\text { de un fenómeno u objeto de estudio. }\end{array}$ & & & & \\
\hline $\begin{array}{l}\text { 8. Identifica ideas, principios, modelos y valores subyacentes en los juicios que se emiten } \\
\text { en las diferentes fuentes de información. }\end{array}$ & & & & \\
\hline $\begin{array}{l}\text { 9. Analiza la congruencia entre sus acciones y los principios y valores que las } \\
\text { fundamentan. }\end{array}$ & & & & \\
\hline 10. Fundamenta críticamente los juicios que se emiten. & & & & \\
\hline $\begin{array}{l}\text { 11. Emite juicios en función de su utilidad, validez y confiabilidad a partir de la información } \\
\text { analizada. }\end{array}$ & & & & \\
\hline \multicolumn{5}{|l|}{ 12. Explica el fenómeno en estudio a partir de sus elementos fundamentales. } \\
\hline \multicolumn{5}{|l|}{ 13. Genera nuevas posibilidades de conocimiento. } \\
\hline 14. Integra los conocimientos previos para el abordaje del fenómeno u objeto de estudio. & & & & \\
\hline \multicolumn{5}{|l|}{ II. Habilidades tecnológicas } \\
\hline 15. Utiliza aplicaciones automatizadas para facilitar la producción de textos digitales. & & & & \\
\hline $\begin{array}{l}\text { 16. Utiliza descriptores, palabras claves y otros filtros para orientar búsquedas de } \\
\text { información en diversos repositorios de información (bases de revistas electrónicas, } \\
\text { sistemas bibliotecarios y otros). }\end{array}$ & & & & \\
\hline \multicolumn{5}{|l|}{$\begin{array}{l}\text { 17. Conoce los diferentes tipos de licencia de los recursos e informaciones digitales y sus } \\
\text { implicaciones en el uso de las mismas. }\end{array}$} \\
\hline 18. Domina algún programa de gestión bibliográfica. & & & & \\
\hline 19. Utiliza algún software para la detección de plagio. & & & & \\
\hline 20. Utiliza algún programa para el análisis de datos. & & & & \\
\hline \multicolumn{5}{|l|}{$\begin{array}{l}\text { 21. Identifica los procesos por seguir para la publicación de artículos en alguna revista } \\
\text { electrónica. }\end{array}$} \\
\hline \multicolumn{5}{|l|}{ III. Habilidades metodológicas } \\
\hline 22. Construye un estado del arte acerca del tema o campo de estudio. & & & & \\
\hline 23. Emplea un sistema de referencias para dar reconocimiento a las fuentes consultadas. & & & & \\
\hline $\begin{array}{l}\text { 24. Utiliza protocolos que evidencien la solicitud y aprobación de autorización sobre } \\
\text { derechos de imagen, participación y uso de información de los sujetos de investigación. }\end{array}$ & & & & \\
\hline $\begin{array}{l}\text { 25. Conduce pertinentemente el proceso de análisis y representación gráfica de la } \\
\text { información. }\end{array}$ & & & & \\
\hline $\begin{array}{l}\text { 26. Plantea problemas de investigación fundamentados en teorias asociadas al estado del } \\
\text { arte construido. }\end{array}$ & & & & \\
\hline $\begin{array}{l}\text { 27. Define preguntas de investigación que conlleven a la posible resolución del problema } \\
\text { planteado. }\end{array}$ & & & & \\
\hline $\begin{array}{l}\text { 28. Propone objetivos de investigación congruentes con las preguntas y el problema de } \\
\text { investigación. }\end{array}$ & & & & \\
\hline $\begin{array}{l}\text { 29. Define el tipo de estudio y selecciona las herramientas metodológicas para alcanzar los } \\
\text { objetivos propuestos. }\end{array}$ & & & & \\
\hline $\begin{array}{l}\text { 30. Interpreta los resultados de la investigación en función de los objetivos y el problema de } \\
\text { investigación. }\end{array}$ & & & & \\
\hline 31. Discrimina y divulga la información relevante derivada del proceso de investigación. & & & & \\
\hline 32. Registra evidencia de la gestión ética y logística del proceso de investigación. & & & & \\
\hline 33. Hace un trato confidencial de la información recolectada en el proceso investigativo. & & & & \\
\hline
\end{tabular}


APÉNDICE 1 (Continuación...)

Escala de autoevaluación de habilidades para la investigación

\begin{tabular}{|c|c|c|c|c|c|}
\hline \multirow{2}{*}{\multicolumn{2}{|c|}{ CRITERIOS POR VALORAR }} & \multicolumn{4}{|c|}{ NIVEL PERCIBIDO } \\
\hline & & \multirow[t]{2}{*}{$\begin{array}{l}\text { Alto } \\
(3)\end{array}$} & \multirow[t]{2}{*}{$\begin{array}{c}\text { Medio } \\
(2)\end{array}$} & \multirow[t]{2}{*}{$\begin{array}{c}\text { Bajo } \\
(1)\end{array}$} & \multirow[t]{2}{*}{$\begin{array}{l}\text { Ninguno } \\
(0)\end{array}$} \\
\hline 34. & Discute los resultados de la investigación a la luz del marco teórico planteado. & & & & \\
\hline 35. & Conoce las formas de atender la validez de las investigaciones cualitativas. & & & & \\
\hline 36. & $\begin{array}{l}\text { Presenta las conclusiones derivadas de la interpretación de los resultados congruentes } \\
\text { con los objetivos de investigación. }\end{array}$ & & & & \\
\hline & $\begin{array}{l}\text { Elabora el reporte de investigación en el que consigne con claridad, el planteamiento del } \\
\text { problema, el marco teórico, los materiales y métodos, los resultados, la discusión de } \\
\text { resultados y las conclusiones y recomendaciones. }\end{array}$ & & & & \\
\hline & $\begin{array}{l}\text { Presenta, en los anexos, la información necesaria para complementar lo descrito en el } \\
\text { reporte de investigación. }\end{array}$ & & & & \\
\hline \multicolumn{6}{|c|}{ IV. Habilidades para gestionar la investigación } \\
\hline & Diseña un proyecto de investigación. & & & & \\
\hline & Dirige un proyecto de investigación. & & & & \\
\hline 41. & Ejecuta planificadamente un proyecto de investigación. & & & & \\
\hline 42. & Conoce diferentes fuentes de financiamiento para investigación. & & & & \\
\hline & Gestiona recursos para la ejecución de proyectos de investigación. & & & & \\
\hline \multicolumn{6}{|c|}{ V. Habilidades para el trabajo en equipo } \\
\hline 44. & Reconoce los aportes y las potencialidades de todos los integrantes del equipo. & & & & \\
\hline 45. & Toma en cuenta los puntos de vista de los demás y realiza críticas constructivas. & & & & \\
\hline 46. & Asume responsablemente las tareas asignadas en su grupo de trabajo. & & & & \\
\hline & $\begin{array}{l}\text { Colabora activamente en la planificación del trabajo en equipo, la distribución de tareas } \\
\text { y la fijación de plazos para asegurar el cumplimiento de los objetivos del proyecto. }\end{array}$ & & & & \\
\hline
\end{tabular}

Fuente: Elaboración propia, 2011. 